\title{
Une carte nationale d'identité biométrique pour les Britanniques : l'antiterrorisme au cœur des discours de justification
}

\section{Laurent Laniel et Pierre Piazza}

\section{(2) OpenEdition \\ Journals}

Édition électronique

URL : http://journals.openedition.org/conflits/2174

DOI : 10.4000/conflits. 2174

ISSN : $1777-5345$

Éditeur :

CCLS - Centre d'études sur les conflits lilberté et sécurité, L'Harmattan

Édition imprimée

Date de publication : 20 décembre 2006

Pagination : 49-63

ISBN : 978-2-296-02667-4

ISSN : 1157-996X

Référence électronique

Laurent Laniel et Pierre Piazza, « Une carte nationale d'identité biométrique pour les Britanniques :

l'antiterrorisme au cœur des discours de justification », Cultures \& Conflits [En ligne], 64 | hiver 2006, mis en ligne le 02 avril 2007, consulté le 30 mars 2021. URL : http://journals.openedition.org/conflits/ 2174 ; DOI : https://doi.org/10.4000/conflits.2174

Ce document a été généré automatiquement le 30 mars 2021

Creative Commons License 


\title{
Une carte nationale d'identité
} biométrique pour les Britanniques : l'antiterrorisme au cœur des discours de justification

\author{
Laurent Laniel et Pierre Piazza
}

1 En Grande-Bretagne, comme dans d'autres pays de Common Law ${ }^{1}$, du fait de la tradition du droit non écrit, toute tentative d'assignation identitaire par l'Etat est historiquement assimilée à une entreprise tyrannique ${ }^{2}$. Lorsque les Britanniques se sont effectivement retrouvés encartés, ce ne fut que pour cause de Seconde Guerre mondiale, période de restriction des libertés et de rationnement alimentaire ${ }^{3}$. Pourtant, à la suite des attentats du 11 septembre 2001, le gouvernement du RoyaumeUni a décidé, à l'instar de ses homologues italien, allemand, espagnol, néerlandais, belge ${ }^{4}$ ou encore français ${ }^{5}$, de faire appel à la biométrie pour encarter ses citoyens, comme en atteste le projet Identity Cards Bill.

2 Matérialisant une initiative du premier cabinet de Tony Blair et constituant l'aboutissement d'un processus gouvernemental de consultation initié dès juillet 2002, ce projet a été adopté le 30 mars 2006 (sous l'appellation «Identity Cards Act 2006 »), après une longue bataille parlementaire opposant le gouvernement à la chambre des Lords, hostile surtout au caractère obligatoire de la nouvelle carte d'identité biométrique. En effet, le projet initial du gouvernement prévoyait d'octroyer cette carte à toute personne demandant l'établissement ou le renouvellement d'un passeport. Or, sachant qu'environ $80 \%$ des Britanniques disposent déjà d'un tel document, les Lords ont estimé que cette mesure, qualifiée pour l'occasion de « contrainte à la dérobée » (compulsion by stealth), rendrait de fait la carte d'identité obligatoire. Le texte finalement adopté stipule que, jusqu'au 1er janvier 2010, les personnes demandant le renouvellement ou l'octroi d'un passeport pourront refuser la carte d'identité. Après cette date, dans le même cas de figure, elles se verront automatiquement attribuer la carte d'identité. Dans tous les cas, les informations 
recueillies sur tous les demandeurs de passeport seront insérées dans le National Identity Register, une base centralisée de données que le gouvernement va constituer sur l'ensemble des résidents du Royaume-Uni à des fins d'identification. A partir de 2008, les permis de résidence accordés aux étrangers seront biométrisés et les données les concernant seront, elles aussi, enregistrées dans cette même base de données. Par ailleurs, le ministre de l'Intérieur Charles Clarke a déclaré, à l'issue de l'adoption de l'Identity Cards Act 2006, qu'il ne doutait pas que, dans l'éventualité d'une victoire de son parti (le parti travailliste) aux prochaines élections générales, le Parlement voterait une loi rendant cette carte d'identité obligatoire pour tous.

3 Pour certains chercheurs, cette entreprise gouvernementale d'encartement biométrique des Britanniques doit être appréciée à l'aune d'une nouvelle forme de coopération transatlantique en matière de sécurité qui se dessine depuis le 11 septembre ${ }^{6}$. D'autres émettent l'hypothèse qu'elle renvoie à l'imposition de modes de gouvernement de plus en plus fondés sur une logique de traçabilité des individus ${ }^{7}$. Plus généralement, comme le souligne Ayse Ceyhan dans le texte introductif de ce numéro de Cultures \& Conflits, cette entreprise s'inscrit dans un contexte global où les préoccupations sécuritaires subissent actuellement de profondes transformations et dans lequel la technologie tend à être érigée en solution la plus appropriée pour faire face à des risques et des menaces présentés comme inédits. Cette "technologisation » de la sécurité pourrait d'ailleurs être replacée dans un contexte historique plus large : celui de l'avènement d'un régime transnational de surveillance de la population issu de la montée, un peu partout dans le monde, de l'insécurité (réelle et perçue) qui a suivi la fin de la Guerre froide. D'après James Sheptycki, l'une des caractéristiques essentielles de ce régime, c'est qu'en se focalisant sur des menaces transnationales qu'il prétend traiter notamment au moyen de la technologie, il détourne l'attention des causes sociales, économiques et politiques, pour la plupart locales ou nationales, de l'insécurité et du sentiment d'insécurité 8 .

4 Mais de quelle manière l'impérieuse nécessité de recourir à la technologie dans le domaine de la sécurité est-elle légitimée par les pouvoirs publics? Comment les arguments qu'ils mettent en avant sont-ils appréciés ? Les réactions suscitées ont-elles un impact sur les discours des gouvernements et leurs stratégies de persuasion ? Force est de constater que, depuis plusieurs années, de nombreuses raisons ont été avancées par les gouvernements britanniques pour faire accepter l'encartement aux citoyens: lutte contre la fraude, hooliganisme, immigration clandestine, etc. Cet article porte sur une autre raison majeure invoquée et sur les variations rhétoriques auxquelles elle a donné lieu depuis l'origine : la lutte contre le terrorisme.

On constate en effet qu'à partir des années 1980, les gouvernements britanniques ont commencé à mettre en avant cet argument dans leurs discours, mais sans jamais véritablement démontrer rigoureusement en quoi une carte nationale d'identité pourrait constituer un instrument efficace de lutte contre le terrorisme. Le caractère peu probant et souvent fragile de cet argument, qui a été de plus en plus mobilisé depuis septembre 2001, constitue l'une des principales explications à l'ampleur des résistances suscitées par le récent projet gouvernemental d'encartement biométrique des citoyens en Grande-Bretagne. Il apparaît donc comme un des facteurs clefs permettant de comprendre les grandes difficultés auxquelles se sont heurtées les instances étatiques pour faire aboutir ce projet. Pourtant, elles y sont progressivement parvenues en infléchissant leur discours qui, en la matière, est devenu à la fois plus 
prudent et plus ambigu, et en faisant valoir le caractère incontournable de prétendues obligations internationales.

La montée en puissance d'une rhétorique officielle peu étayée

6 A partir du milieu des années 1980, l'argument terroriste, au même titre que d'autres, est de plus en plus systématiquement évoqué à chaque fois qu'est discuté au Parlement et dans la presse un projet visant à instaurer un dispositif d'encartement des Britanniques. Ainsi, en 1985, une proposition de loi émanant du député conservateur John Biggs-Davison recommande l'institution d'une carte nationale d'identité en vue de rendre plus efficace le combat mené par les autorités contre l'IRA. L'élu tory n'apporte cependant alors aucun détail sur la façon dont la diffusion de ce document pourrait effectivement aider les pouvoirs publics dans la lutte antiterroriste ${ }^{9}$. D'ailleurs, un examen approfondi des débats parlementaires de cette époque montre que cette imprécision caractérise tous les propos des députés se réclamant de cet argument. C'est toujours le cas, quatre ans plus tard, dans le cadre du National Identity Card Bill présenté par Ralph Howell (député conservateur). Pour convaincre ses collègues que la carte d'identité dont il envisage la création serait précieuse pour faire face au terrorisme, il évoque de manière allusive deux événements: le crash aérien de Lockerbie et le fait que deux journalistes déguisés en membres du personnel de nettoyage soient parvenus à pénétrer sans autorisation dans un avion stationné à l'aéroport de Heathrow. Sommé de préciser son propos, Howell répond prudemment qu'il ne souhaite pas épiloguer sur cet attentat et se contente d'indiquer que, grâce à sa carte d'identité, les entreprises sauront dorénavant plus précisément qui elles emploient. Il indique ensuite que ce titre permettrait surtout de combattre une criminalité qualifiée de " problème encore plus important que le terrorisme ${ }^{10}$ ». Aucun des députés conservateurs favorables à sa proposition de loi ne déroge au flou qui est alors de mise. Tout au plus, Roy Beggs, seul parlementaire à faire explicitement état du terrorisme irlandais, déclare-t-il :

«l'absence d'une carte nationale d'identité permet à des individus aux intentions criminelles de traverser, sans entrave aucune, le pays dans l'anonymat le plus complet et avec la quasi-certitude de ne jamais rencontrer le moindre obstacle ${ }^{11}$.

On ne trouve pas davantage de précisions dans les déclarations officielles formulées au cours des années 1990, décennie durant laquelle les initiatives favorables à la mise en place de procédures d'encartement des citoyens établissent pourtant un lien plus direct avec le terrorisme nord-irlandais. En mars 1991, le député David Trimble (chef de file des loyalistes d'Ulster) essaie d'introduire dans le projet de loi Northern Ireland Bill, présenté à la chambre des Communes, une clause prévoyant l'institution d'une carte d'identité obligatoire qu'il considère comme absolument nécessaire au nom de la lutte contre le terrorisme en Irlande du Nord. Mais en vain, car, de l'aveu même de certains membres ou alliés du gouvernement conservateur de l'époque, cette initiative est bien peu rigoureusement justifiée au vu des critiques argumentées qu'elle engendre. Ainsi, Lord d'Arran, ministre de l'Irlande du Nord de John Major, déclare devant la chambre des Lords: "Les forces de sécurité ne considèrent pas que l'identification pose problème. L'instauration de cartes constituerait un fardeau bureaucratique très lourd pour tout le monde». Dans la même enceinte parlementaire, Lord Ferrers (un conservateur proche du gouvernement) ajoute qu'il ne peut se souvenir d'un seul acte terroriste susceptible d'avoir pu être évité par la création d'une carte d'identité obligatoire, avant de préciser que «le gouvernement examine tous les moyens possibles pour empêcher le terrorisme dans notre pays, et les cartes d'identité ne sont 
pas prioritaires à cet égard ${ }^{12}$ ». Ce revers n'empêche pas David Trimble de tenter une nouvelle fois sa chance devant la chambre des Communes lors d'un débat consacré, en mars 1994, au renouvellement du Prevention of Terrorism Act, adopté en 1989. Mais son discours va rester tout aussi vague puisqu'il se contente de souligner qu'une carte nationale d'identité s'avèrerait particulièrement utile afin d'accroître la surveillance de la population ${ }^{13}$. De même, toujours aux Communes, le 22 juin 1994, lors de la présentation de sa proposition de loi relative à l'institution d'une carte d'identité, le député conservateur Harold Elletson déclare, sans étayer plus avant son propos, que le fait, pour les propriétaires de logements à court terme ${ }^{14}$, de pouvoir vérifier efficacement qui sont leurs locataires grâce à une carte d'identité représenterait une contribution très importante à la lutte antiterroriste ${ }^{15}$.

8 Aucune de ces initiatives établissant un lien direct entre l'impératif de mise en œuvre d'un dispositif d'identification des citoyens par le papier et les besoins de la lutte antiterroriste ne s'est concrétisée. Les raisons d'un tel échec renvoient à des résistances ancrées dans des registres contestataires variés: coûts financiers induits par les entreprises d'encartement, crainte d'une détérioration des relations entre police et population, risques de bureaucratisation accrue, etc. ${ }^{16}$. Néanmoins, un autre facteur mérite d'être sérieusement pris en considération : les promoteurs de ces initiatives ne sont jamais parvenus à expliquer avec clarté et précision comment une carte nationale d'identité permettrait d'améliorer la lutte contre le terrorisme. Si bien que, dans son Livre vert sur les cartes d'identité ${ }^{17}$, rendu public en mai 1995, le gouvernement dirigé par John Major est amené à légitimer prudemment le nouveau dispositif d'identification qu'il propose alors. Son Livre vert fera surtout référence à ses bienfaits supposés en matière de lutte contre la criminalité et la question terroriste sera reléguée au rang de légitimant secondaire.

$9 \mathrm{Au}$ lendemain des attentats du 11 septembre, les autorités britanniques ont constamment eu recours à l'argument de la lutte antiterroriste pour expliquer combien leur projet de carte nationale d'identité électronique contenant des données biométriques s'avérait indispensable. Lorsqu'elles ne se sont pas contentées de faire référence au terme générique de "terrorisme " pour indiquer qu'il renvoyait à une menace au même titre que l'» immigration clandestine » ou la " criminalité organisée », ces autorités ont généralement repris à leur compte les raisons avancées quelques années plus tôt. Ainsi, pour le ministre de l'Intérieur David Blunkett, la nouvelle carte d'identité biométrique constitue un « moyen simple et sécurisé de vérifier les identités ${ }^{18}$ ", c'est-à-dire, à ses yeux, un remède à la fraude identitaire, précieux pour combattre des terroristes censés recourir à de fausses identités pour se jouer de toute forme de contrôle.

Un argumentaire qui donne prise à critiques et résistances

10 L'emploi de fausses identités par les terroristes est une réalité qui ne saurait évidemment être niée. Pour autant, comme a pu le souligner une étude rendue publique par Privacy International en avril $2004{ }^{19}$, il n'est aucunement démontré que l'existence d'une carte nationale d'identité constitue une mesure antiterroriste efficace. De nombreux passages de cette étude ont été repris dans un long rapport intitulé The Identity Project rédigé par la London School of Economics en mars 2005, portant sur le projet de loi de carte d'identité biométrique. Stigmatisant notamment la sousestimation gouvernementale du coût financier de son dispositif d'encartement (officiellement estimé à 3,1 milliards de livres sterling sur dix ans en 2002, puis à 5,5 
milliards en novembre 2004), ce rapport cite aussi les propos du Secrétaire général d'Interpol, Ron Noble, qui n'a pas hésité, fin 2004, à affirmer devant la chambre des Lords que tous les cas de terrorisme impliquait l'usage d'un faux passeport, sans pourtant pouvoir apporter quelque preuve que ce soit pour étayer cette assertion ${ }^{20}$.

11 Comme avait déjà pu l'expliquer à la chambre des Communes, en février 1989, le député travailliste Gary Waller (Keighley) lors de la discussion susmentionnée de la proposition de loi de carte d'identité du député Ralph Howell ${ }^{21}$, en signalant toutefois combien les libertés publiques pourraient en souffrir, une carte nationale d'identité pourrait revêtir une utilité incontestable dans la lutte contre le terrorisme si les informations qu'elle permet de se procurer sur les détenteurs de ce titre (l'ensemble des citoyens dans le cas où ce document deviendrait obligatoire) étaient interconnectées avec d'autres données personnelles détenues par les autorités à des fins administratives, fiscales, policières ou judiciaires et une multitude de renseignements exploités à des fins de surveillance. Dans un tel cas de figure, le fichier informatique centralisé, élaboré grâce à l'ensemble des données recueillies sur les porteurs d'une carte, constituerait un instrument supplémentaire d'un vaste dispositif technologique autorisant non seulement le repérage d'individus préalablement identifiés comme dangereux, mais aussi l'élaboration, dans le cadre des analyses de la menace terroriste, de profils à risque. Or, de manière récurrente, le gouvernement britannique souligne que son projet actuel de mise en carte des citoyens permet de sécuriser utilement l'identité de chacun et ne s'inscrit nullement dans une logique susceptible de porter atteinte aux libertés individuelles.

12 Si la biométrisation de la carte d'identité renvoyait in fine à un tel objectif de surveillance généralisée au nom de la lutte contre le terrorisme, les risques encourus seraient non négligeables eu égard à l'efficacité réelle des mesures d'encartement préconisées. Ainsi, selon le sociologue canadien David Lyon, le projet britannique de carte d'identité «intelligente» aurait surtout pour conséquence d'augmenter la capacité des instances étatiques à se livrer à un «tri social » en discriminant certaines catégories de la population au comportement stigmatisé comme indésirable ${ }^{22}$. Au-delà de ces craintes, se pose également le problème de la production de discours policiers sur l'anticipation des comportements terroristes qui ne reposent sur aucune forme de savoir scientifique. En effet, pour d'autres chercheurs, ce type de discours a pour seule fonction de justifier l'introduction de dispositifs « high tech » dont pourtant rien ne garantit qu'ils seront opérationnels pour combattre le terrorisme ${ }^{23}$. C'est dans cette logique que semblent s'inscrire les propos du ministre de l'Intérieur David Blunkett. En 2004, il a par exemple pu affirmer que la carte d'identité ne permettrait pas d'arrêter les terroristes mais qu'elle jouerait un rôle préventif facilitant grandement le travail des services antiterroristes ${ }^{24}$. Pourtant, la même année, certains policiers de haut rang soulignaient combien il apparait vain d'essayer de combattre sérieusement des terroristes avec une carte nationale d'identité. Ainsi, Stella Rimington, ancienne directrice générale du MI5 (service de contre-espionnage britannique), a pu déclarer qu'une telle carte «ne permettrait aucunement de rendre le pays plus sûr et que dans l'éventualité d'une falsification, ce document ne servirait absolument plus à rien ${ }^{25}$ ».

Il est vrai que les dispositifs d'encartement des citoyens sont loin d'apparaitre comme la solution sécuritaire la plus appropriée pour faire face, par exemple, aux nouvelles formes de terrorisme islamiste radical. C'est d'ailleurs l'idée que n'hésitent pas à avancer, en France - où pourtant la première «carte d'identité des Français » a été 
instituée dès $1921^{26}$-, certains anciens hauts responsables de la lutte antiterroriste. Ainsi, pour Jean-Pierre Pochon (DCRG, DST, DGSE), ce type de mesure technologique sophistiquée ne peut avoir qu'un intérêt relatif car il ne peut suffire seul à empêcher certains individus déterminés de commettre un acte terroriste : le plus important pour les services de renseignement est de pouvoir repérer ces mêmes individus préalablement, alors que la carte d'identité ne servira généralement qu'à établir leur identité une fois l'attentat commis ${ }^{27}$.

De plus, au vu des événements récents, les terroristes peuvent se recruter au sein même de la communauté des citoyens britanniques. Dès lors, en quoi une carte nationale d'identité, extrêmement fiable ou non, pourrait-elle être d'une quelconque utilité pour combattre des terroristes à même d'obtenir ce document en toute légalité ? $\mathrm{Au}$ Canada, ce type d'argument est d'ailleurs aussi systématiquement avancé par les opposants à l'introduction d'une carte nationale d'identité. Le Centre de recherche et d'information sur le Canada (CRIC) en résume la teneur :

15 «La manière précise dont ces cartes d'identité nationales permettraient de lutter contre le terrorisme demeure vague. La comparaison de nos cartes d'identité nationales aux listes de surveillance des terroristes connus ou soupçonnés s'avérerait inutile lorsqu'il s'agit de nouveaux terroristes inconnus ayant recours à des documents d'identification légitimes. L'établissement d'une énorme base de données avec toutes nos transactions quotidiennes, qui pourrait par la suite être explorée dans la recherche d'indices liés au terrorisme, serait hautement envahissante et irait à l'encontre de nos traditions de common law et de la protection de nos libertés civiles bien établies ${ }^{28}$ ».

On constate en effet que, lors des attentats commis à Londres en juillet 2005, certains terroristes disposaient de papiers en bonne et due forme qui ont d'ailleurs été retrouvés sur les lieux des explosions ${ }^{29}$ : comme si ces individus tenaient de la sorte à afficher et rendre visible la spécificité de leur statut comme un ultime défi aux valeurs de l'Etat dont ils étaient ressortissants.

17 Cet exemple montre à quel point il apparaît illusoire d'envisager de combattre efficacement des terroristes à la fois membres d'une communauté nationale et ennemis idéologiques de celle-ci, au moyen d'un outil renvoyant exclusivement à l'un des deux termes de l'équation : l'appartenance nationale. C'est le constat qu'a notamment dressé la chambre des Lords dans un avis daté du 16 décembre 2004 : les citoyens peuvent se révéler tout aussi dangereux pour la sécurité du pays que les ressortissants étrangers ${ }^{30}$.

18 Le caractère superficiel et souvent fragile de la justification étatique des projets d'encartement des citoyens par la lutte antiterroriste explique dans une large mesure les fortes oppositions qu'ils suscitent depuis les années 1980 en Grande-Bretagne. Il est au cœur des résistances qui émanent d'acteurs de natures très différentes: citoyens isolés, hauts fonctionnaires de police, associations, organes de presse et partis politiques. De plus, ces résistances s'expriment à travers des registres d'action de plus en plus variés. Ce sont bien sûr de traditionnelles polémiques qui prennent forme dans les médias et les arènes parlementaires. Le ministre de l'Intérieur Charles Clarke a, par exemple, sévèrement critiqué dans la presse le caractère "partial » du rapport de la London School of Economics de mars 2005 qui concluait à l'inutilité du projet gouvernemental de carte nationale d'identité biométrique en matière antiterroriste. Ses propos ont aussitôt été réfutés dans le Times (2 juillet 2005) par le directeur de cette célèbre école et dans le Daily Telegraph (7 juillet 2005) par vingt-deux chercheurs ayant tenu à affirmer publiquement leur soutien aux rédacteurs de ce rapport. Voyant 
dans l'opposition à la carte d'identité une opportunité de se démarquer nettement des deux principaux partis politiques, les Libéraux démocrates - la troisième force politique du pays - ont fait leur l'argument de l'inefficacité de la carte dans la lutte antiterroriste. On le trouve d'ailleurs en bonne place dans une rubrique de leur site web spécifiquement consacrée à cette question ${ }^{31}$. A côté d'un formulaire permettant de signer une pétition en ligne demandant l'abandon du projet gouvernemental de mise en carte, il est rappelé que les cartes d'identité obligatoires en Espagne n'ont pas empêché les attentats du 11 mars 2004 à Madrid.

La mobilisation contre le projet d'encartement du gouvernement britannique emprunte donc aussi massivement le canal d'Internet. Des sites web de contestation entièrement consacrés à cet enjeu ont été créés par des associations qui insistent sur le caractère abusif du lien établi entre antiterrorisme et mise en carte des citoyens. Par exemple, le site Internet de l'association Sayno2ID ${ }^{32}$ pose la question : "Comment la carte d'identité nous protégera-t-elle de la menace terroriste?», et y répond catégoriquement: "Elle ne nous en protègera pas ${ }^{33}$ ». Le même type d'argument est développé sur pratiquement tous les sites Internet des grandes ONG britanniques spécialisées dans la défense des droits de l'Homme et des libertés individuelles. Liberty va même jusqu'à mettre en ligne un petit livret intitulé ID Cards - Fiction and Fact, qui constitue un recueil d'arguments contre le projet gouvernemental de carte d'identité au sein duquel la thèse antiterroriste est la première à être réfutée ${ }^{34}$.

Infléchissement de la stratégie discursive du gouvernement

Le caractère fragile de l'argumentation des pouvoirs publics britanniques a donc donné du poids à la contradiction et placé les responsables des projets de mise en carte dans une posture délicate ${ }^{35}$. Face à cette situation, le poids des contraintes internationales (normes adoptées par l'OACI en matière de données biométriques ${ }^{36}$, nouvelles formes de coopération instituées entre l'Union européenne et les Etats-Unis en ce qui concerne l'échange de données personnelles et l'instauration du passeport biométrique par l'Union européenne ${ }^{37}$ ) a constitué pour le gouvernement Blair un argument auquel il a de plus en plus recouru pour convaincre l'opinion publique du caractère judicieux de son entreprise de mise en carte. A cet égard, le Premier ministre lui-même a plusieurs fois puisé dans le registre de l'évidence ou de la fatalité, affirmant par exemple :

«Il s'agit d'avoir une longueur d'avance [a step ahead] ${ }^{38}$ sur les terroristes [...]. De toute façon nous allons bien devoir suivre les évolutions des exigences techniques américaines et européennes vers l'adoption de passeports biométrisés. Le coût supplémentaire que représente l'introduction de cartes d'identité est parfaitement justifié eu égard à la plus-value qui en résultera en matière de sécurité ${ }^{39}$ ».

21 Il a ainsi pu suggérer l'idée que la biométrisation de la carte d'identité était devenue quasiment inéluctable, notamment $\mathrm{du}$ fait de l'adoption de certaines normes européennes, alors même que le règlement européen du 13 décembre 2004 relatif à l'introduction d'éléments biométriques dans les titres de voyage ne concernait nullement les cartes d'identité (voir son article 1, alinéa $3{ }^{40}$ ).

La ressource supranationale a aussi été mobilisée en partenariat avec les principaux pays européens désireux de recourir à la biométrie pour mettre en carte leurs citoyens. C'est ainsi qu'à la réunion du G5 qui s'est déroulée à Evian les 4 et 5 juillet 2005, les ministres de l'Intérieur britannique, français, espagnol, italien et allemand ont convenu d'œuvrer en faveur de la délivrance de cartes d'identité électroniques compatibles et «interopérables». En outre, ils ont proposé d'étendre la biométrie à tous les documents d'identification. Ce processus avait d'ailleurs été impulsé deux mois plus tôt 
par les ministres de l'Intérieur des mêmes pays. En mai 2005, ils co-signaient une tribune publiée par Le Figaro dans laquelle on pouvait lire :

«A cinq, nous avons décidé d'aller plus loin ensemble en travaillant sur des cartes nationales d'identité électroniques compatibles: des programmes sont engagés conjointement par la France et l'Allemagne, d'autres sont développés par l'Italie, l'Espagne et le Royaume-Uni ${ }^{41}$ ».

Cette stratégie a été de plus en plus fréquemment mise en œuvre par les autorités britanniques qui n'ont pas hésité à faire appel à un registre discursif désormais "classique »: l'invocation de la nécessité de respecter les obligations européennes... quitte à les créer soi-même lorsque le moment apparaît politiquement opportun. Ainsi, le 13 juillet 2005, une semaine à peine après les attentats de Londres, Charles Clarke a présidé une session extraordinaire du Conseil Justice et Affaires intérieures (JAI) réunissant l'ensemble des ministres de l'Intérieur et de la Justice des vingt-cinq pays membres de l'UE. La déclaration condamnant les attentats émise à l'issue du Conseil demandait aux Etats-membres d'adopter des «normes communes pour les éléments de sécurité et des procédures sûres de délivrance des cartes d'identité », et ce, afin de lutter contre le terrorisme ${ }^{42}$. Quatre mois plus tard, cette même justification est réapparue dans une note de la présidence britannique de l'Union européenne adressée aux ministres de l'Intérieur des pays membres. La note en question accompagnait un projet de "conclusions" relatives aux normes minimales à adopter en matière de sécurisation des dispositifs d'encartement des citoyens. Lesdites "conclusions" résultaient des réflexions d'un « groupe d'experts ad hoc » dont ni la composition ni les règles de fonctionnement ne sont rendues publiques. Relevant d'une "soft law" souvent stigmatisée comme illustrant le déficit démocratique de l'Union européenne ${ }^{43}$, elles ont pourtant été adoptées lors du Conseil des ministres de l'Intérieur de l'UE en date des 1er et 2 décembre 2005, présidé par Charles Clarke ${ }^{44}$.

Dans le même temps, soumis à une forte pression médiatique à l'heure où la GrandeBretagne est l'un des derniers pays européens à ne pas disposer de carte nationale d'identité, le gouvernement a plusieurs fois été contraint de présenter l'encartement des citoyens comme n'étant pas la panacée. Ainsi, en novembre 2004, lors de sa conférence de presse mensuelle, Tony Blair a finalement dû expliquer à propos de la carte :

«Bien entendu, elle ne représente pas la solution miracle contre le terrorisme et la criminalité organisée, personne ne dit cela, mais elle constituera une arme importante dans la lutte contre les menaces modernes que posent le terrorisme et la criminalité organisée ${ }^{45} »$.

Même si, sur le fond, le Premier ministre n'a pas apporté de précisions supplémentaires permettant de mieux comprendre en quoi la carte serait une « arme importante » pour s'attaquer au terrorisme, sa posture montre à quel point il est apparu soucieux de contrer les critiques auxquelles s'expose son projet. En s'évertuant à ne pas ériger la carte en remède idéal face au terrorisme, tout en indiquant, parallèlement, que cet instrument peut tout de même se révéler particulièrement utile en la matière, Tony Blair entend désamorcer toutes les contestations que serait susceptible d'amplifier une argumentation étatique trop simpliste ou monofactorielle. Ces dernières années, cette stratégie a été récurrente. En avril 2004, à une question lui enjoignant de préciser si le gouvernement considérait la carte nationale d'identité avant tout comme un instrument de lutte antiterroriste, le porte-parole du Premier ministre répondait prudemment par la négative en indiquant que ce document avait une large gamme 
d'utilité, même si « la lutte antiterroriste est une question qu'il ne faut pas sous-estimer ${ }^{46}$ ». De même, deux mois plus tard, le porte-parole de Tony Blair a souligné de nouveau que le fait d'apporter des arguments complémentaires pour montrer combien la carte s'avère indispensable n'amoindrissait en rien celui de la prévention du terrorisme. Et, à une interpellation d'un journaliste lui faisant remarquer que le terrorisme avait "glissé » en bas de la liste des raisons mises en avant par le gouvernement pour légitimer l'instauration de ce titre, il rétorquait : « La liste pourrait tout aussi bien être lue dans l'autre sens, cela n'a pas d'importance, toutes ces raisons sont de bonnes raisons pour se doter de cartes d'identité ${ }^{47} \ggg$. Est-ce ainsi qu'il faut aussi lire la liste des motifs évoqués par les pouvoirs publics dans la loi finalement adoptée le 30 mars 2006 (Identity Cards Act 2006) afin de justifier, au nom de "l'intérêt général» (public interest), la nécessité d'instaurer un National Identity Register? Dans le cadre de cette loi, l'» intérêt général » est restreint à cinq domaines de l'action publique :

«(a) Dans l'intérêt de la sécurité nationale ;

(b) dans l'objectif de prévenir ou de détecter les faits délictueux;

(c) dans l'objectif de faire respecter la législation applicable en matière de contrôle de l'immigration; (d) dans l'objectif de faire respecter les interdictions édictées en matière de travail au noir ; ou

(e) dans l'objectif de garantir la mise en œuvre efficace et effective de services publics ${ }^{48}$ ".

On constate que la lutte antiterroriste ne fait pas partie des arguments explicitement mis en avant dans la loi, le terme plus englobant de "national security " lui ayant été apparemment préféré. Le contenu même de ce texte législatif semble constituer l'aboutissement d'une stratégie gouvernementale désormais plus habile, qui allie prudence et ambiguïté. En effet, les termes de "sécurité nationale» et «faits délictueux » renvoient, sans le nommer directement, à celui de "terrorisme ", comme le spécifient d'ailleurs, pour ceux qui n'auraient pas compris, les «notes explicatives » accompagnant l'Identity Cards Act $2006{ }^{49}$.

Les gouvernements conservateurs et travaillistes du Royaume-Uni invoquent, depuis trente ans, l'impératif de la lutte antiterroriste pour imposer leurs projets " high-tech » d'encartement des Britanniques. De telles initiatives sont à l'origine de larges mouvements contestataires, notamment parce que leurs promoteurs n'ont pas réussi à démontrer clairement que la carte nationale d'identité peut constituer une arme antiterroriste. Non seulement leurs argumentaires n'ont pas convaincu, mais, en outre, ils ont prêté le flanc à une multitude de critiques.

Si le gouvernement actuel est parvenu à faire voter la loi instituant la carte d'identité biométrique, c'est au prix d'un net infléchissement de son discours qui introduit désormais une distance entre l'objectif antiterroriste et le dispositif d'identification. Dès lors, on peut se demander pourquoi les pouvoirs publics se sont efforcés de lier l'enjeu de l'encartement des citoyens à celui de la lutte contre le terrorisme avec autant d'insistance, alors même que le « remède » suggéré semble inadapté à la « maladie ». A cet égard, la réflexion mériterait de s'orienter dans deux directions. La première concerne le rôle joué par les outils technologiques ultra-sophistiqués dans la valorisation des réponses que les politiques proposent face au terrorisme. Si de tels outils peuvent contribuer à accroître la visibilité de leur action, surtout en temps de crise, on peut aussi penser qu'ils permettent effectivement de rassurer un grand nombre de citoyens, ne serait-ce qu'au titre de placebo agissant sur le sentiment d'insécurité - mais au prix d'un renforcement du savoir et du pouvoir policiers. La 
seconde a trait aux multiples enjeux stratégiques auxquels renvoient les technologies de pointe. Des recherches approfondies portant sur les aspects économiques, industriels et financiers des applications biométriques permettraient d'analyser plus finement les ressorts de la décision politique et de la rhétorique qui l'accompagne.

\section{NOTES}

1.. Sur le cas australien, voir notamment Greenleaf G., Nolan J., « The deceptive history of the Australia card », Australian Quarterly, 58, (4), 1987. Pour une synthèse des critiques que suscitent chez les « libertariens » les initiatives visant à instaurer une carte nationale aux Etats-Unis, voir Etzioni A., « Big Brother or big benefits? », Les Cahiers de la sécurité, $n^{\circ} 56,1 \mathrm{er}$ trimestre 2005, pp. 31-37. Toutes les citations de cet article ont été traduites de l'anglais par les auteurs.

2.. Voir notamment sur cet aspect Pimentel C., «L'exception britannique, l'identité non écrite », in Crettiez X., Piazza P. (dir.), Du papier à la biométrie. Identifier les individus, Paris, Les Presses de Sciences Po, 2006.

3.. La carte d'identité a été instaurée en Grande-Bretagne par une ordonnance du 11 septembre 1939 qui sera abrogée le 21 février 1952. Pour plus de détails sur ce dispositif de mise en carte des citoyens et sur le processus ayant conduit à son abandon, voir notamment Agar J., « Modern horrors: British identity and identity cards », in Caplan J., Torpey J. (dir.), Documenting Individual Identity. The Development of State Practices in the Modern World, Princeton, Princeton University Press, 2001 et Dovey H.O., " Why national registration had to go ", Public Administration, Londres, n64, hiver 1986.

4.. Pour une présentation générale des projets d'encartement biométrique des citoyens initiés par les gouvernements de ces différents pays, voir :

http://www.cnil.fr/fileadmin/documents/approfondir/dossier/CNI-biometrie/identiteelectronique-EUROPE.pdf

5.. Sur la nature du projet INES (Identité nationale électronique sécurisée) et les résistances qu'il suscite, voir la contribution de Pierre Piazza dans ce numéro.

6.. Voir Guiraudon V., « La coopération transatlantique après le 11 septembre : l'enjeu de la sécurité intérieure ", Critique internationale, n²8, juillet-septembre 2005, p. 34.

7.. Lyon D., Surveillance After September 11, Oxford, Polity Press, 2003.

8.. Voir Sheptycki J., En quête de police transnationale. Vers une sociologie de la surveillance à l'ère de la globalisation, coll. « Perspectives criminologiques », Larcier/ De Boeck, Bruxelles, 2005, en particulier pp. 173-213.

9.. Voir l'article « Time to bring in identity cards » qu'il publie dans le Times du 7 février 1985.

10.. Sur le débat parlementaire consacré à la proposition de loi de Ralph Howell, voir : http://www.publications.parliament.uk/cgibin/newhtml_hl?

$\mathrm{DB}=$ semukparl\&STEMMER=en\&WORDS=national\%20identity\%20card\%20bill\&ALL=\&ANY=\&PHRASE=\%22National\%20Iden pa/cm198889/cmhansrd/1989 02 10/Debate 1.html\#Debate 1_spnew42; citations

column 1271

11.. Ibid. 
12.. Ces citations sont extraites de Thomas P.A., « Identity cards », Modern Law Review, 58 (5), septembre 1995, pp. 707-708.

13.. http://www.publications.parliament.uk/cgibin/newhtml_hl?

$\mathrm{DB}=$ semukparl\&STEMMER

$=$ en $\&$ WORDS=trimbl\%20card\&ALL=Trimble\%20 $\mathrm{card} \& A N Y=\& P H R A S E=\& C A T E G O R I E S=\& S I M P L E=\& S P E A K E R=\& C O L O U R=$ red $\& S T Y L E=s \& A N$ $\mathrm{pa} / \mathrm{cm} 199394 / \mathrm{cmhansrd} / 1994-03-09 /$ Debate-3.html\#Debate-3_spnew29, column 315

14.. C'est-à-dire les hôtels et les bed and breakfasts.

15.. http://www.publications.parliament.uk/cgibin/newhtml_hl?

$\mathrm{DB}=$ semukparl\&STEMMER

en\&WORDS=elletson\&ALL=Elletson\&ANY=\&PHRASE=\&CATEGORIES=\&SIMPLE=\&SPEAKER=\&COLOUR=red\&STYLE=s\&ANCH pa/cm199394/cmhansrd/1994-06-22/Debate-1.html\#Debate-1_spnew18, column 239

16. Sur ces différents points, voir notamment Redfern P., « A population register or identity cards for 1992 ", Public Administration (Londres), n68, hiver 1990.

17. Home Office, Identity Cards: a consultation document, CM 2879, mai 1995. Un « Livre vert » (Green paper) est un document gouvernemental visant à instaurer un débat sur un problème de société particulier auquel il se propose de remédier, ou encore, comme c'était le cas en l'occurrence, qui est destiné à tester l'opinion sur telle ou telle mesure dont le gouvernement envisage l'application.

18. Commentaire de David Blunkett sur le rapport du Select Committee de la chambre des Communes relatif au projet de carte d'identité biométrique, « Home secretary sets out next steps on ID cards », Home Office Press Releases, 27 octobre 2004, http://press.homeoffice.gov.uk/press-releases/Home_Secretary_Sets_Out_Next_Ste?version=1 19.. Privacy International, Mistaken Identity: Exploring the Relationship Between National Identity Cards \& The Prevention of Terrorism, avril 2004 : http:// www.privacyinternational.org/issues/idcard/uk/id-terrorism.pdf 20.. London School of Economics \& Political Science, The Identity Project. An assessment of the UK Identity Cards Bill \& its implications, Londres, mars 2005, p. 32 : http://is.lse.ac.uk/idcard/identityreport.pdf

21.. Voir http://www.number-10.gov.uk/output/page7758.asp

22.. Lyon D., « La frontière est partout : encartement, surveillance et altérité. Réflexions autour du projet anglais de carte d'identité intelligente », Les Cahiers de la sécurité, $\mathrm{n}^{\circ} 56,1$ er trimestre 2005.

23. Voir par exemple les propos tenus par Didier Bigo lors de son audition par la CNIL sur le projet français de carte nationale d'identité biométrique : http://www.cnil.fr/ fileadmin/documents/approfondir/dossier/CNI biometrie/CRAUDITIONBIGO.pdf

24.. Cité dans Privacy international, «ID cards "will not stop terrorism" ", The Guardian, 27 avril 2004.

25.. Cité dans White M., Glover J., « Government fights on as peers inflict defeats on ID card bill », The Guardian, 17 janvier 2006.

26.. Voir Piazza P., « Septembre 1921 : la “première carte d'identité des Français” et ses enjeux », Genèses, n54, mars 2004.

27. Entretien réalisé par les auteurs avec Jean-Pierre Pochon, 8 juillet 2005. Un citoyen britannique avance le même type d'argument qu'il résume en une phrase sur sa page web personnelle dans laquelle il s'oppose à l'entreprise d'encartement conduite par son gouvernement : «Le meilleur service que puisse rendre une carte d'identité c'est de prouver l'identité de quelqu'un. Mais elle ne permettra jamais de prouver ses intentions. Si je me réveille demain matin avec l'idée de poser une bombe, aucune carte 
d'identité ne pourra m'en empêcher ", http://www.trevor-mendham.com/civilliberties/identity-cards/fight-crime.html

28.. http://www.cric.ca/fr_html/guide/national_id_card/id_card.html\#contre

29.. Il s'agissait de permis de conduire, voir notamment BBC, « Suicide

bombers"'ordinary" lives », http://news.bbc.co.uk/1/hi/uk/4678837.stm

30.. http://www.parliament.the-stationeryoffice.co.uk/pa/ld200405/ldjudgmt/ jd041216/a\&others.pdf

31.. http://www.libdems.org.uk/noidcards/

32.. C'est-à-dire « Say no to ID » qui signifie « Dites non à la carte d'identité ».

33.. http://www.no2id.net/IDSchemes/FAQ/problemsSolved.php\#terrorism

34.. http://www.liberty-human-rights.org.uk/privacy/id-cards-fiction-and-fact.shtml

35.. Outre les réactions d'hostilité et résistances évoquées précédemment, la chambre des Lords a refusé quatre fois le projet gouvernemental d'encartement. Fin janvier 2006, le Joint Committee on Human Rights du Parlement avait notamment émis de sérieuses réserves quant à la compatibilité de l'Identity Cards Bill avec les articles 8 et 14 de la Convention européenne des droits de l'Homme. Voir United Kingdom Parliament, Joint Committee on Human Rights- Fifth Report, 2 février 2006 : http://www.publications.parliament.uk/pa/jt200405/jtselect/jtrights/35/3503.htm 36.. Sur ce point, voir Ceyhan A., "Identité et identification au prisme de la biométrie ", intervention au séminaire de philosophie du droit organisé par l'IHEJ (« Sécurité, sûreté, surveillance »), 20 mars 2006, p. 5 : http://www.ihej.org/ressources/ ceyhan_20_03_06_2006.pdf

37.. Sur ces deux derniers points, voir notamment Mitsilegas V., " Contrôle des étrangers, des passagers, des citoyens : surveillance et anti-terrorisme », Cultures \& Conflits, $\mathrm{n}^{\circ} 58,2005$, pp.155-181.

38. Tony Blair faisait ici référence au livre blanc intitulé One Step Ahead. A 21st Century Strategy to Defeat Organised Crime, que son gouvernement avait publié un an plus tôt. Sur ce point, voir Sheptycki J., « Dans la machine construite sur la criminalité transnationale. Réflexions sur les transformations des paysages policiers nationaux ", Les Cahiers de la sécurité, n57, 2e trimestre 2005.

39.. Press briefing avec le porte-parole officiel du Premier ministre, 25 mai 2005 : http://www.pm.gov.uk/output/page7548.asp. Rappelons que les «évolutions des exigences techniques américaines et européennes » auxquelles fait allusion le Premier ministre britannique découlent elles-mêmes des recommandations formulées par l'Organisation de l'aviation civile internationale - l'OACI, résolution en date du 9 mai 2003 - en matière de biométrisation des documents de voyage.

40.. Règlement CE, $\mathrm{n}^{\circ} 2252 / 2004$.

41.. « Une Europe plus sûre, une Europe plus solidaire », Le Figaro, 12 mai 2005.

42.. Conseil de l'Union européenne : «Communiqué de presse, Session extraordinaire, Justice et Affaires intérieures », Press Office, Bruxelles, 13 juillet 2005, p. 7. La présidence britannique du Conseil de l'UE avait évoqué, deux jours plus tôt, la nécessité d'instaurer ces mêmes « normes communes de sécurité » dans le cadre d'une note consacrée à l'immigration et aux frontières de l'Union où il n'était aucunement question de lutte antiterroriste. Voir Council of the European Union, Note from the Presidency to Strategic Committee on Immigration, Frontiers and Asylum on Minimum Common Standards for National Identity Cards, Bruxelles, 11 juillet 2005.

43.. Non contraignante, la " soft law » est également non soumise à l'approbation des parlements nationaux. Sur le rôle joué par la «soft law » et les " groupes d'experts » 
dans la prise de décision de l'Union européenne en matière de sécurité, voir de Maillard J., Smith A., Gouverner la sécurité : entre Etats-nations et Europe, Convention de recherche INHES ( $\left.n^{\circ} 02113\right)$, juillet 2005.

44.. Council of the European Union, Press Release, 2696th Council Meeting, Justice and Home Affairs, Bruxelles 1er et 2 decembre 2005, 14 390/05 (presse 296), pp. 34-35.

45.. Conférence de presse mensuelle du Premier ministre, 29 novembre 2004, voir : http://www.number-10.gov.uk/output/page6687.asp

46.. Press briefing du porte-parole du Premier ministre, 26 avril 2004, voir : http://www.number-10.gov.uk/output/page5704.asp

47.. Rencontre du porte-parole du Premier ministre avec la presse, 29 juin 2005, voir : http://www.number-10.gov.uk/output/page7758.asp

48.. Identity Cards Act 2006, chapter 15, 1, (4), p. 2 :

http://www.opsi.gov.uk/acts/acts2006/ukpga_20060015_en.pdf

49.. Identity Cards Act 2006, Explanatory Notes, 18, p. 4 :

http://www.opsi.gov.uk/acts/en2006/ukpgaen_20060015_en.pdf

\section{RÉSUMÉS}

A partir des années 1980, les gouvernements britanniques ont commencé à mettre en avant l'argument de la lutte antiterroriste dans leurs discours visant à justifier le besoin d'instaurer une carte nationale d'identité dans leur pays. Mais ils ne sont jamais parvenus à démontrer rigoureusement comment un tel document permettrait de lutter efficacement contre le terrorisme. Le caractère peu probant et souvent fragile de l'argumentaire antiterroriste, davantage mobilisé depuis le 11 septembre 2001, constitue une des principales explications à l'ampleur des résistances suscitées par l'Identity Cards Bill, projet gouvernemental d'encartement biométrique des citoyens britanniques lancé en 2002. En dépit de grandes difficultés, les autorités sont finalement parvenues à faire aboutir ce projet en 2006, mais en infléchissant leur discours, devenu à la fois plus prudent et plus ambigu, et en faisant valoir le caractère incontournable de prétendues obligations internationales.

In the 1980s, British governments started using the argument of the fight against terrorism in order to justify the need to adopt a national identity card in the UK. Yet, they have never succeeded in demonstrating indisputably that ID cards may help fight terrorism. The unfounded and unconvincing nature of this governmental rhetoric is one of the main reasons for the strong resistance in the UK against the Identity Cards Bill, the project of biometric ID cards for British citizens launched by the government in 2002. In spite of many difficulties, the government eventually succeeded, and the bill became law in 2006, but only after adapting its rhetoric, which became both more prudent and more ambiguous, and by alleging the incontrovertibility of socalled international obligations. 
INDEX

Mots-clés : anti-terrorisme, bases de données, biométrie, identité

Index géographique : Royaume Uni

Index chronologique : 2001 post 11 septembre

\section{AUTEURS}

\section{LAURENT LANIEL}

Laurent Laniel est sociologue, chargé de recherche à l'INHES. Auteur de nombreux articles et traductions sur le trafic international des drogues illicites, il a récemment publié avec P.-A. Chouvy « Production agricole de drogues illicites et conflictualités intra-étatiques : dimensions économiques et stratégiques » (Cahiers de la sécurité, $n^{\circ} 62$, 3e trimestre 2006). Il anime le site DrugStrat (http://laniel.free.fr).

\section{PIERRE PIAZZA}

Pierre Piazza est maître de conférences en science politique à l'université de Cergy-Pontoise. Il a notamment publié Histoire de la carte nationale d'identité (Paris, Odile Jacob, 2004) et dirigé, avec Xavier Crettiez, Du papier à la biométrie. Identifier les individus, Paris, Les Presses de Science Po, 2006. 